\title{
Physical stability of model emulsions based on ethyl cellulose oleogels
}

\author{
Iwona Szymańska1(1)*, Anna Żbikowska1 ${ }^{1}$, and Małgorzata Kowalska² \\ ${ }^{1}$ Department of Food Technology and Assessment, Warsaw University of Life Sciences (WULS-SGGW), \\ Nowoursynowska 166, 02-787 Warsaw, Poland \\ ${ }^{2}$ Faculty of Chemical Engineering and Commodity Science, Kazimierz Pulaski University of Technology and Humanities, \\ Chrobrego 27, 26-600 Radom, Poland
}

Received March 3, 2020; accepted May 12, 2020

\begin{abstract}
The purpose of this study was the evaluation of the physical stability of low-fat oil-in-water model emulsions containing oleogels based on ethyl cellulose), compared to emulsions with anhydrous milk fat. The oleogels were prepared using rapeseed oil with ethyl cellulose at the level of 4.5, 5.0, 5.5 or $6.0 \% \mathrm{w} / \mathrm{w}$. The $\mathrm{O} / \mathrm{W}$ emulsions $(30 / 70 \mathrm{w} / \mathrm{w})$ were stabilized by guar gum $(0.6 \% \mathrm{w} / \mathrm{w})$ and soy lecithin $(5.0 \% \mathrm{w} / \mathrm{w})$. The physical properties of oleogels and anhydrous milk fat (centrifugal stability, spreadability/penetration test), rheology and the physical stability of the emulsions (MS-DWS method, centrifugal/thermal stability) were determined, a storage test of the emulsions (CSA method, LUMiSizer) was also conducted. It was demonstrated that ethyl cellulose is an effective edible oil structuring agent. The increase in ethyl cellulose content enhanced the centrifugal stability and hardness of the oleogels. As a result of a microrheology analysis, it was found that the emulsion with anhydrous milk fat had the most elastic strength, macroscopic viscosity and the highest solids content. All emulsions demonstrated high centrifugal/ thermal stability. A higher temperature and extended storage time caused a reduction in the stability of all emulsions and increased the velocity of particle migrations. The ethyl cellulose-oleogels are potential fat phases for stable $\mathrm{O} / \mathrm{W}$ emulsions, which could be used as a vegan alternative to traditional products based on animal components.
\end{abstract}

Keywords: oleogels, ethyl cellulose, oil-in-water emulsions, MS-DWS method, storage test, CSA method

\section{INTRODUCTION}

The nutritional values and technological properties of fat depend on the composition of their fatty acids and also their distribution in triacylglycerol molecules. Fat that

*Corresponding author e-mail: iwona_szymanska@sggw.edu.pl remains solid at room temperature contains a significant amount of saturated fatty acids (SFA), it has the greatest utility value which is due to the stable structure of the final product (Orsavova et al., 2015). According to an EFSA report, SFA intake, regardless of its origin, should be as low as possible (EFSA, 2017).

Due to the presence of cholesterol, the milk fat present in food, over $60 \%$ of which is SFA and which is sold at a relatively high price, is being replaced by palm fat - which is fat without cholesterol, it is relatively inexpensive and naturally solid at room temperature, but contains more than $50 \%$ SFA. Rapeseed oil is characterized by an optimal composition of fat content: approx. 7\% SFA, 60\% monounsaturated fatty acids (MUFA) and 30\% polyunsaturated fatty acids - including approx. 10\% $\alpha$-linolenic acid (Lindman, 2014; Dybkowska, 2015). In order to obtain fats with desirable properties, various methods of modification (structuring) are used (Ferreira et al., 2019). Currently, scientists are interested in intensive activities aimed at eliminating partially hydrogenated and animal fats, i.e. research concerning oleogelation. It is a new technique with great potential in the production of structural lipids (Ögütcü et al., 2015; Onacik-Gür et al., 2019). It enables the formation of three-dimensional structures of lipid gels as a result of the gelation of vegetable oils with a structuring agent of lipid origin (e.g. waxes, monoacylglycerols) or polysaccharide origin (e.g. ethyl cellulose) (Hughes et al., 2009). Ethyl cellulose (EC) is a water-insoluble cellulose derivative that

(C) 2020 Institute of Agrophysics, Polish Academy of Sciences 
can be plasticized, this is partially because it mixes easily with oils and waxes (Żbikowska et al., 2019). According to Żbikowska et al. (2017), ethyl cellulose is one of the most effective polymer oleogelators and it can be used in food production.

Many food products, that contain different types of oils/ fats, are emulsions. The oil-in-water emulsions, as heterogeneous multi-component systems, are a good matrix for use in laboratory tests because they are considered to be equivalent to complex foods. The properties of the emulsion depend significantly on the choice of raw materials, recipe composition, technological process and storage conditions (Kowalska et al., 2017; Szymańska et al., 2020). With regard to the concept of "emulsion instability", destabilization mechanisms are classified as reversible (e.g. sedimentation, flotation) or irreversible (e.g. flocculation, coalescence). These phenomena can occur in a relatively complex way, and the course of their development is not observed during a visual assessment. Traditional methods only allow for an estimation of the overall durability of the systems and also for the quantification of the final result of the analysis (Piotrowska and Waszkiewicz-Robak, 2007; Kowalska and Żbikowska, 2016). Stability studies using various methods have been described in the subject literature, however as yet, there are still no papers that simultaneously use the CSA method (LUMiSizer dispersion analyser) and the MS-DWS method (Rheolaser Master) to test systems in terms of their physical stability.

In relation to the above, oleogels can be used to partially or completely replace conventional solid fats in food. The oleogelation process is based on a different principle than the well-known methods of modifying fats, e.g. fractionation, hydrogenation or transesterification. It allows for fat to be obtained from oil which has a high content of essential unsaturated fatty acids, without reducing its health value (Öğ̈̈tcü and Yılmaz, 2014). The application of oleogels (with an optimal composition of FA) instead of animal or vegetable fats (supplying TFA and SFA) in widely consumed products, including those based on high or low-fat emulsions, may contribute to decreasing the risk of cardiovascular diseases. In view of the fact that oleogels are potentially highly stable fat systems, oil migration may be limited and the durability of food products may be increased (Co and Marangoni, 2012; Stortz et al., 2012).

As yet, there is no information in the literature concerning the possibility of obtaining stable $\mathrm{o} / \mathrm{w}$ emulsions in which the fat phase is an oleogel. The novelty of the study is the replacement of solid animal fat with highly nutritional solid vegetable fat obtained by oleogelation. Modern stability testing techniques (LUMiSizer, Rheolaser Master) were used to check the properties of the dispersion systems.

The aim of the study was to assess the possibility of using ethyl cellulose oleogels to obtain low-fat oil-in-water emulsions and to determine the variations in their physical stability over time.

\section{MATERIALS AND METHODS}

Ethyl cellulose (EC) structured oleogels and emulsions were analysed. The control samples were anhydrous milk fat (SM Mlekovita, Poland) - AMF (99.8\% milk fat, including $65 \%$ SFA; $0.1 \%$ protein) and emulsion obtained with AMF - emulsion $\mathrm{K}$. The following raw materials and additives were used: refined rapeseed oil $-7.5 \%$ SFA, $65.5 \%$ MUFA, 26.5\% PUFA, including 8\% PUFA from the $\omega-3$ group (Kruszwica S.A., Poland), ethyl cellulose EC45 premium (Dow Inc., USA) as a structuring agent, guar gum (Hortimex, Poland) as a thickener, soy lecithin (Cargill Inc., USA) as an emulsifier, sodium benzoate as a preservative, and distilled water. All of the ingredients were used within the period of their shelf-life.

Initial tests established a minimum EC level of $4.5 \%$ $\mathrm{w} / \mathrm{w}$ per $100 \mathrm{~g}$ of oleogel. The oleogel samples with an EC content ranging from $1 \%$ to $4 \%(\mathrm{w} / \mathrm{w})$ were semi-liquid at room temperature and had an oil-binding capacity below $70 \%$.

Rapeseed oil with ethyl cellulose - EC (4.5, 5.0, 5.5, $6.0 \% \mathrm{w} / \mathrm{w}$ per $100 \mathrm{~g}$ of oleogel) were heated on a single burner electric stove Adler AD 6503 (ADLER Sp. z o.o., Poland) at $140^{\circ} \mathrm{C}$ for $45 \mathrm{~min}$, and mixed at $600 \mathrm{r} \mathrm{min}^{-1}$ using electric stirrer JJ-1B (Wincom Company Ltd., China). The temperature was controlled using a portable thermocouple. The samples were set aside on the laboratory countertop for $2 \mathrm{~min}$, and after that, they were heated (at $120^{\circ} \mathrm{C}$ ) and mixed (at $400 \mathrm{r} \mathrm{min}^{-1}$ ) for $15 \mathrm{~min}$. If the mixture was not clear, the last step was repeated.

The compositions of the model emulsions were chosen according to preliminary research and scientific papers (Kowalska et al., 2014; Szymańska et al., 2020). The fat phase was prepared from AMF (control sample - K) or oleogel (EC 4.5/ EC 5.0/ EC 5.5/ EC 6.0). The amounts of emulsifier (soy lecithin) and thickener (guar gum) were constant -5.0 and $0.6 \% \mathrm{w} / \mathrm{w}$ accordingly. Sodium benzoate $(0.25 \% \mathrm{w} / \mathrm{w})$ was used as a preservative. Variable amounts of water were determined by the content of the other ingredients and the need to obtain an equal mass for all of the samples $-100 \mathrm{~g}$ (Table 1). The volume of the aqueous phase was $70 \% \mathrm{w} / \mathrm{w}$ for each emulsion.

The process of preparing the emulsion is shown in Fig. 1. Both the aqueous and fatty phase (with AMF or EC oleogels) were prepared using a magnetic stirrer Phoenix RSM 10-HP with rotational speed and temperature control (Phoenix Instrument GmbH, Germany). The homogenization of the aqueous phase and fatty phase was carried out by using an ULTRA-TURRAX T25 homogenizer (IKA Labortechnik, Germany). All of the samples were maintained at $20 \pm 1^{\circ} \mathrm{C}$ for $24 \mathrm{~h}$ prior to the following measurements.

The oleogel samples were submitted to the effect of centrifugal forces. Approximately $3 \mathrm{~g}$ of oleogel or AMF was centrifuged in a centrifuge MPW-352 (MPW Med. 
Table 1. Composition of emulsions based on AMF or EC-oleogels

\begin{tabular}{|c|c|c|c|c|c|c|}
\hline \multirow{2}{*}{$\begin{array}{l}\text { Type } \\
\text { of sample }\end{array}$} & \multirow{2}{*}{$\begin{array}{l}\text { Fat } \\
\text { phase }\end{array}$} & \multicolumn{5}{|c|}{ Component $(\%, \mathrm{w} / \mathrm{w})$} \\
\hline & & Fat & $\begin{array}{c}\text { Soy } \\
\text { lecithin }\end{array}$ & $\begin{array}{l}\text { Guar } \\
\text { gum }\end{array}$ & $\begin{array}{c}\text { Sodium } \\
\text { benzoate }\end{array}$ & $\begin{array}{c}\text { Distilled } \\
\text { water }\end{array}$ \\
\hline K & $\mathrm{AMF}$ & & & & & \\
\hline E I & $\mathrm{EC} 4.5$ & & & & & \\
\hline E II & EC 5.0 & 30 & 5 & 0.6 & 0.25 & up to 100 \\
\hline E III & EC 5.5 & & & & & \\
\hline E IV & EC 6.0 & & & & & \\
\hline
\end{tabular}

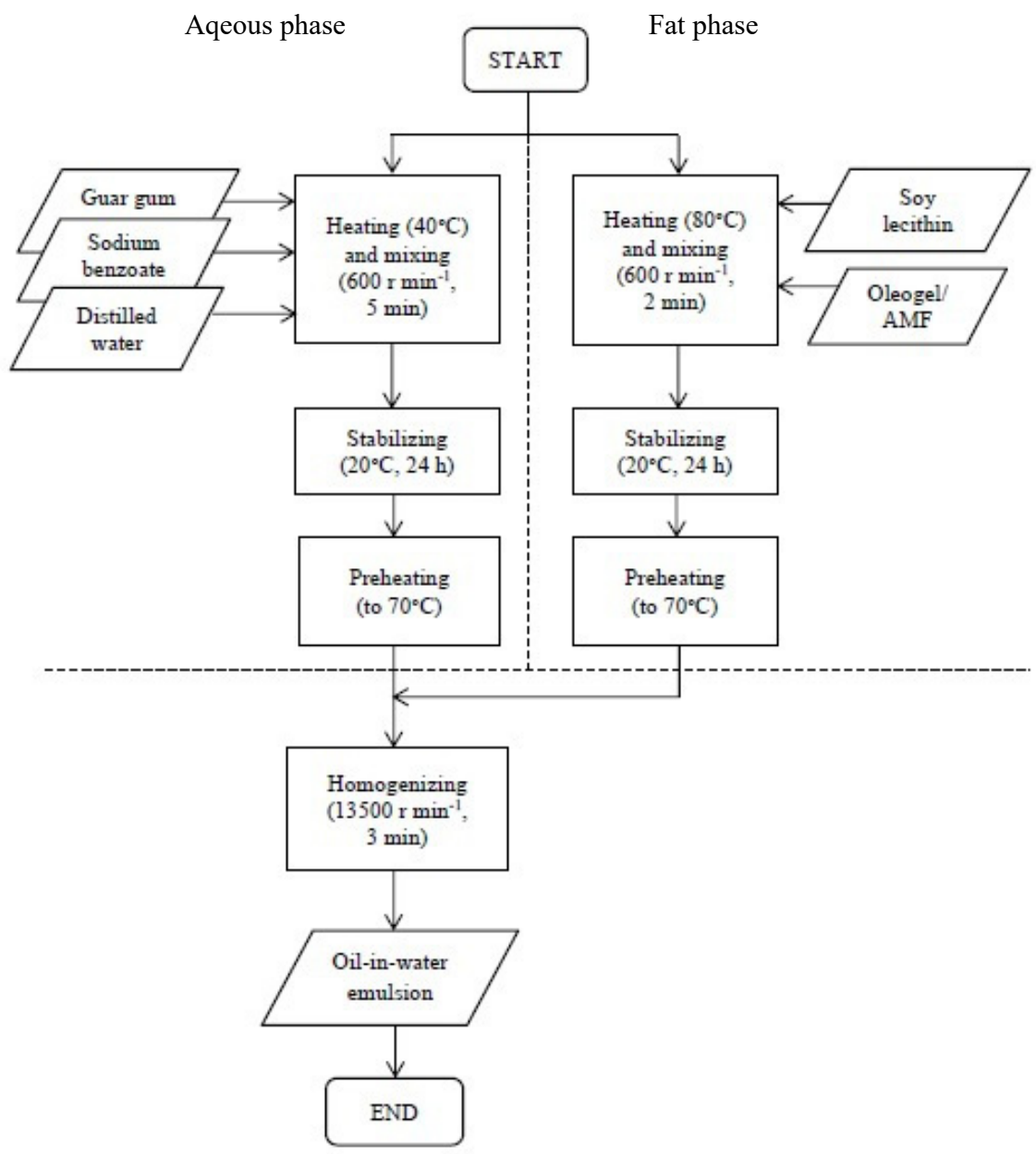

Fig. 1. Block diagram of obtaining model emulsions.

Instruments, Poland) at $4000 \mathrm{r} \mathrm{min}{ }^{-1}$ for $15 \mathrm{~min}$. If the lipid liquid phase was separated from oleogel or AMF, it was relatively unstable. After centrifugation, the tubes were inverted for $30 \mathrm{~min}$ to remove the released oil. The oil binding capacity (centrifugal stability) of oleogels/AMF was calculated based on the following equation (Öğ̈̈tcü and Yılmaz, 2014; Onacik-Gür et al., 2017):

$$
O B C=\frac{M_{a}-M_{t}}{M_{b}-M_{t}} \quad 100,
$$

where: $O B C$ - oil binding capacity or centrifugal stability (\%, mass), $M_{a}-$ weight of oleogel/AMF and test tube after centrifugation without the released oil $(\mathrm{g}), M_{b}$ - weight of oleogel and test tube before centrifugation $(\mathrm{g}), M_{t}$ - weight of the test tube $(\mathrm{g})$.

The samples were analysed in a penetration test, using a TX.AT plus (Micro Stable Systems, UK). A working component with a cylindrical tip $(\mathrm{P} / 0.5 \mathrm{R})$ with a diameter of $10 \mathrm{~mm}$ was used. During the analysis, this tip was moved into the sample at a rate of $1 \mathrm{~mm} \mathrm{~s}^{-1}$ to a depth of $5 \mathrm{~mm}$. 
The sample was approximately $30 \mathrm{~g}$ of oleogel in a $50 \mathrm{ml}$ glass beaker. The maximum force measured was defined as hardness $(\mathrm{N})$ and the minimum force as stickiness $(\mathrm{N})$. The test was carried out in six replicates at $23 \pm 1{ }^{\circ} \mathrm{C}$.

Oleogels and AMF were subjected to a spreadability test using a TX.AT plus device (Micro Stable Systems, UK). The TTC Spreadability Rig (HDP/SR fixture) comprised a male $90^{\circ}$ cone probe and precisely matched female perspex cone shaped product holders. The sample of oleogel or AMF was added with a spatula to the lower cone holders and then the surface was levelled. The samples were penetrated by the movable male cone at a speed of $3 \mathrm{~mm} \mathrm{~s}^{-1}$ to a depth of $23 \mathrm{~mm}$, then it returned to its original position at a speed of $10 \mathrm{~mm} \mathrm{~s}^{-1}$. Spreadability was expressed by the work of shear - area under the time-force curve $(\mathrm{N} \mathrm{mm})$ in the range of positive values, and adhesiveness by the work of adhesion - area over the time-force curve $(\mathrm{N} \mathrm{mm})$ in the range of negative values. These parameters were determined using the Exponent software (Micro Stable Systems, UK). The spreadability test was carried out in triplicate at $23 \pm 1^{\circ} \mathrm{C}$.

The microrheology of the emulsions were determined using a Rheolaser Master ${ }^{\mathrm{TM}}$ device (Formulaction, L'Union, France), whose operation is based on the dynamic multi-speckle diffusing-wave spectroscopy technique (MS-DWS). The sample is illuminated by a coherent laser beam $(650 \mathrm{~nm})$, which is scattered by any encountered particles, and a multi-pixel detector (CCD camera, $27 \mathrm{~Hz}$ ) detects backscattered light during measurement (speckles images). The Brownian motions of the particles are measured, which corresponds to the viscoelastic properties of the sample. The measurement results are recorded using the Rheotest software, in the form of curves representing the values of the Mean Square Displacement (MSD, $\mathrm{nm}^{2}$ ) over time, i.e. the average distance covered by the particles in the tested sample volume. The Elasticity Index (EI, $\mathrm{nm}^{-2}$ ), Solid-Liquid Balance (SLB, $\mathrm{nm}^{2}$ ) and Macroscopic Viscosity Index (MVI, $\mathrm{nm}^{-2}$ ) were determined based on the MSD curves obtained. The EI value is an inverse of the MSD, and corresponds to the elastic modulus at the plateau. The SLB value is a solid-liquid balance ratio, and its changes are correlated with the MSD (at plateau) slope at short decorrelation times. SLB $>0.5$ means that the liquid behaviour of the sample dominates and SLB $<0.5$ indicates that there are more solid portions. The MVI parameter presents the macroscopic viscosity at zero shear. It is computed using the inverse of the slope value, and illustrates the viscosity changes (Pasqua et al., 2014; Liu et al., 2020). The emulsions were tested $24 \mathrm{~h}$ after they were obtained. The samples were transferred in $20 \mathrm{ml}$ flat bottom vials, and then the "quick characterization" test was carried out at $22 \pm 1^{\circ} \mathrm{C}$ for $10 \mathrm{~min}$ (in triplicate for each sample).

The centrifugal stability of the model emulsions was determined by centrifuge MPW-352 (MPW Med. Instruments, Poland). The samples (5 g) were centrifuged at $3000 \mathrm{r} \mathrm{min}^{-1}$ for $30 \mathrm{~min}$ (at $23 \pm 2^{\circ} \mathrm{C}$ ). When phase separation was not observed, it was inferred that the emulsion was stable (Kowalska et al., 2014; Szymańska et al., 2020).

The emulsions $(5 \mathrm{~g})$ were subjected to heating at $100 \pm 2{ }^{\circ} \mathrm{C}$ for $30 \mathrm{~min}$. Then the model dispersions were cooled to room temperature $\left(20 \pm 1^{\circ} \mathrm{C}\right)$ and centrifuged at $1815 \mathrm{r} \mathrm{min}^{-1}$ for 5 min using centrifuge MPW-352 (MPW Med. Instruments, Poland). The value of the emulsion stability was the mass ratio of the intact sample to the total mass of the emulsion. If no changes were observed, the sample was assumed to be $100 \%$ stable (Szymańska et al., 2020).

In order to determine the changes in overall stability and the kinetics of destabilization of the model emulsions, a storage stability test was carried out under three temperature conditions (at 4,20 and $40^{\circ} \mathrm{C}$ ). The emulsions were tested after 1, 7, 14, 21 and 28 days of storage using a CSA method (Centrifugal Stability Analysis) - the LUMiSizer 612 dispersion analyser (LUM GmbH, Germany). An accelerated stability test enables the early detection of phenomena occurring and the tracking of particle movement during destabilization. Undiluted samples are subjected to a centrifugal force (centrifugal separation) and illuminated with near infrared light (NIR) - light transmission by the samples is recorded in the form of profiles illustrating the kinetics of the demulsification process. Using a test lasting several hours, it is possible to quickly estimate dispersion stability, this would take several months/years under natural conditions. The test provides information concerning instability mechanisms, changes in particle characteristics, velocity in the spin field or instability indexes (Xu et al., 2017; Zielińska, 2018).

The following test parameters were used: centrifugal force $\left(4000 \mathrm{r} \mathrm{min}^{-1}\right)$, light factor (1.0), wavelength $(870 \mathrm{~nm})$, temperature $\left(20 \pm 1^{\circ} \mathrm{C}\right)$, transmission profiles recording time (10 s), total duration of analysis (50 $\mathrm{min})$. The recorded transmission profiles ("fingerprints") were converted into extinction profiles, which illustrate the intensity of light reflected by the sample - a high smooth curve indicates the full stability of the system. A local reduction in profile height is associated with a decrease in particle concentration, which means a lower extinction coefficient (higher light transmission). This is mainly due to the migration of the particles. As a result of the analysis, the light intensity distribution profiles as a function of time and location along the entire sample were obtained. In addition, the instability indexes and the velocity of the particles in the centrifugation field were calculated, also, the types and intensity of the destabilization phenomena of the emulsions were determined (SEPView software). A stability analysis was carried out in triplicate for each variant.

The analysis of variance was performed using a one-way ANOVA test. Significant differences between the mean values (level of significance: $p<0.05$ ) were determined using a Fisher's test. Homogeneous groups were obtained in the 
Duncan test. In addition, a cluster analysis was performed, which consisted of the classification of the tested emulsions (objects), according to the average values of the determined parameters (variables). For this purpose, the agglomeration method (object classification method), Euclidean distance (distance between objects), Ward method (cluster/object binding principle), Baker and Hubert index (the number of clusters) were used. The affiliation of objects to clusters were tested, for which the average variable values and coefficients of variation were determined. A statistical analysis was performed using the Statistica 13.3 software (TIBCO Software Inc., Palo Alto, CA, USA).

\section{RESULTS AND DISCUSSION}

The macroscopic properties of oleogels depend primarily on the concentration of the edible oil structuring agent (Lupi et al., 2011). Scientific research has proven that an increase in oleogelator content (regardless of its type) improves the ability of the system for oil retention and provides a more solid structure (Öğ̈̈tcü and Yılmaz, 2014; Onacik-Gür and Żbikowska, 2019). A similar dependence was demonstrated for oleogels based on rapeseed oil and ethyl cellulose (Fig. 2). Żbikowska et al. (2019) proved that the oil-binding capacity $(\mathrm{OBC})$ value is an indicator of the stability of the analysed lipids. It has been shown that the examined emulsions differ statistically significantly in terms of OBC values, depending on the amount of oleogelators used. The increase in ethyl cellulose concentration from 4.5 to $6.0 \%$ contributed to an increase in the centrifugal stability value by approx. 19\% (Fig. 2). The control sample was an anhydrous milk fat. In the centrifugal test, the oil binding capacity is sometimes assumed to be $100 \%$, in the case of this type of fat (Co and Marangoni, 2012; Öğütcü and Y1lmaz, 2015; Onacik-Gür et al., 2017). However, in this study the AMF was subjected to a centrifugal test. The

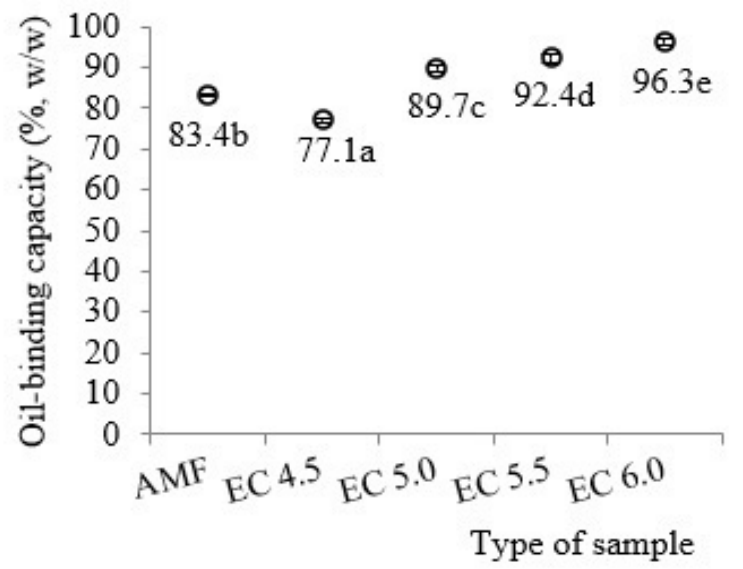

Fig. 2. Oil-binding capacity/centrifugal stability of anhydrous milk fat (AMF) and EC-oleogels. a, b, c - different letters mean statistically significant differences.
OBC for the control sample was $83.3 \%$, and the separated liquid fraction was treated as the unbound lipid portion. The intact solid phase was a stable part of the sample, as were the oleogel samples after centrifugation (Fig. 2). This proceeding was considered to be justified because changes in the physical properties of the fat over time are associated with the phenomenon of migration, which in turn affects the stability of the structure of the target product containing this fat. It was found that only EC 4.5 oleogel was less stable $(77.1 \%)$ than the control sample (83.3\%).

The textural and rheological properties of oleogels (e.g. creaminess, softness, plasticization, aeration, etc.) determine their further application (Öğ̈̈tcü and Y1lmaz, 2014; Onacik-Gür and Żbikowska, 2019). The structure of the oleogel depends both on the concentration of the structuring agent and on the parameters of oleogelation, as well as on the type of oil. Laredo et al. (2011) and Zetzl et al. (2012) reported that oleogel hardness is correlated with the degree of unsaturation of the oil. Flaxseed oil oleogel (above 50\% n-3 fatty acids) has a higher packing density of molecules, compared to rapeseed oil oleogel (approx.10\% n-3 FA) at the same ethyl cellulose content. Laredo et al. (2011) tried to explain the mechanism of formation of oleogels at the molecular level and proved that oil is a passive filler in the gel matrix and does not affect the mechanical properties of the gel through chemical interactions. Figure 3 shows the hardness and stickiness of oleogels based on rapeseed oil with different EC levels. It was found that a $6 \% \mathrm{EC}$ content resulted in a penetration force of approx. $0.80 \mathrm{~N}$ and a work of shear of approximately $6.29 \mathrm{~N} \mathrm{~mm}$. These values were more than three times lower than they were for anhydrous milk fat. In addition, the control sample demonstrated a greater stickiness/adhesiveness, as opposed to the oleogels (Figs 3 and 4). As the EC content increased, the oleogel developed a higher hardness value and lower spreadability. Żbikowska et al. (2019) came to similar conclusions. The use of $7 / 8 \%$ ethyl cellulose as an oleogelator enabled the formation of a structure with a penetration force of $0.6 / 1.8$ $\mathrm{N}$ and work of shear of $25.6 / 51.0 \mathrm{~N} \mathrm{~mm}$ respectively. In the studies of Zetzl et al. (2012) and Gravelle et al. (2013) the dependence of the mechanical properties of oleogels on the concentration and viscosity of ethyl cellulose was taken into account. The greater EC viscosity means a higher molecular weight of polymer chains and an increase in the amount of EC affects the formation of a more integrated and stabilized lipid network.

It was found that structuring the rapeseed oil with ethyl cellulose using the new oleogelation technique allows for the original fat system to be obtained with comparable or greater stability, in comparison with anhydrous milk fat.

Anhydrous milk fat and oleogels based on ethyl cellulose $(4.5 / 5.0 / 5.5 / 6.0 \% \mathrm{w} / \mathrm{w})$ were used as the fat phases of oil-in-water model emulsions $(30 / 70 \mathrm{v} / \mathrm{v})$. The elasticity index (EI), solid-liquid balance (SLB) and macroscopic viscosity index (MVI) were determined using the MS-DWS 

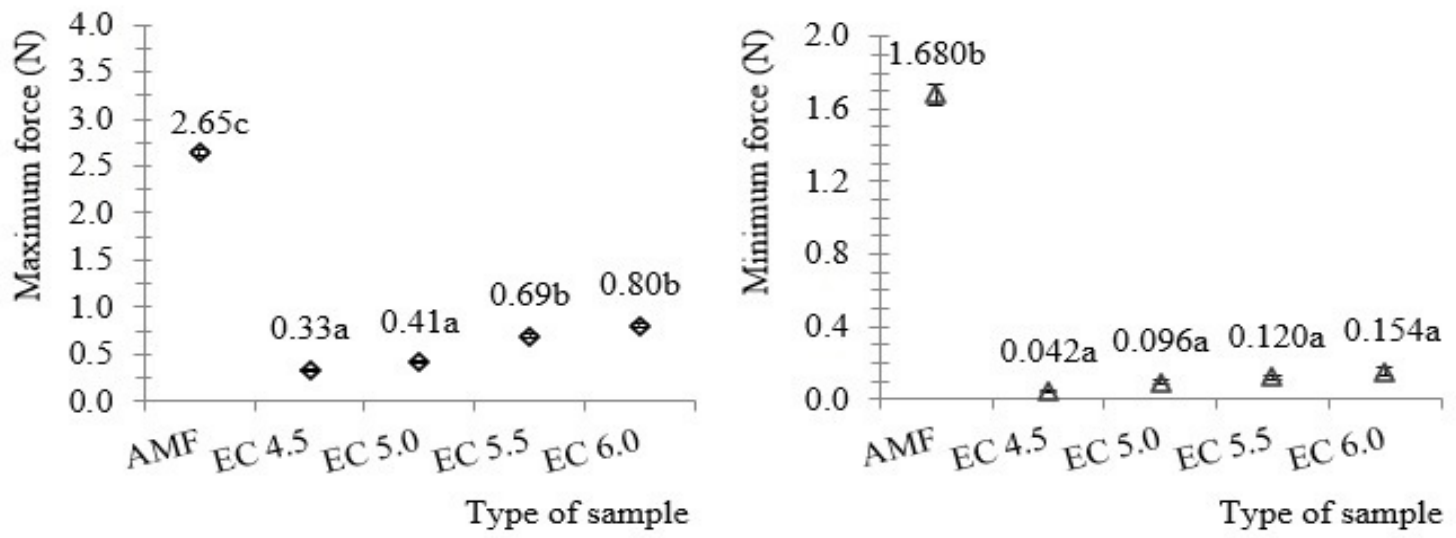

Fig. 3. Penetration test results of anhydrous milk fat (AMF) and EC-oleogels. a, b, c - different letters mean statistically significant differences.
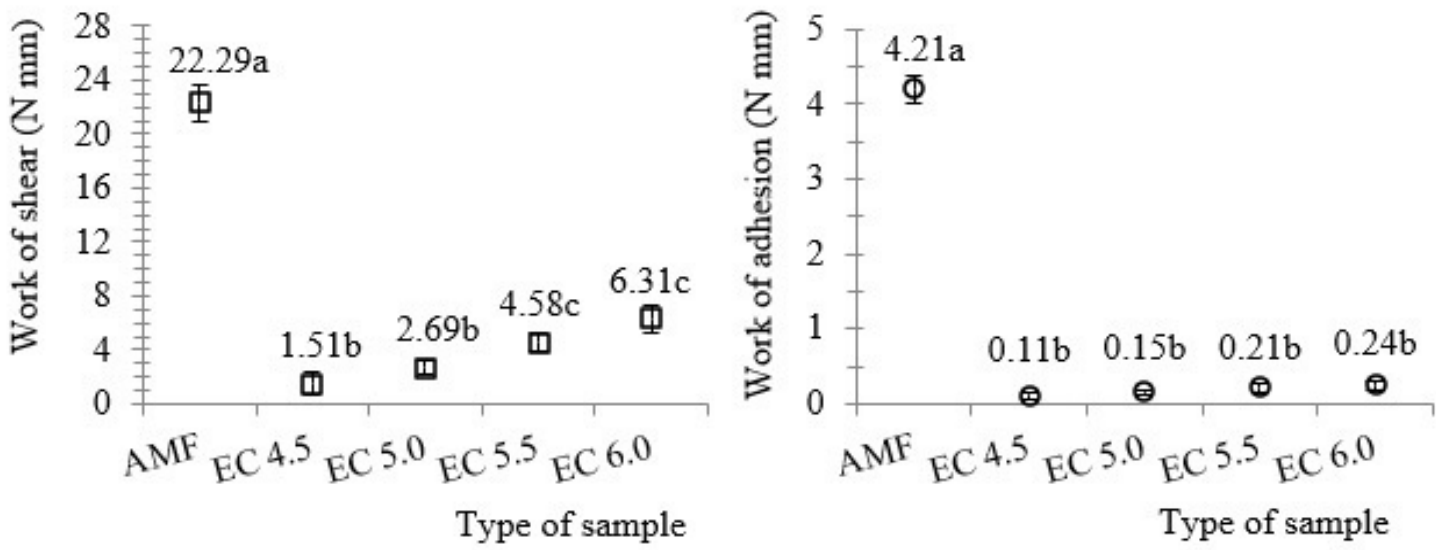

Fig. 4. Spreadability test results of anhydrous milk fat (AMF) and EC-oleogels. a, b, c - different letters mean statistically significant differences.

method. The MS-DWS technique allows for the non-contact measurement parameters of product microrheology at rest (no mechanical/external stress) to be determined. The designated values may be used to compare physically similar products (Tisserand et al., 2011).

It was noted that the AMF-based emulsion (sample K) has an approximately $30 \%$ higher EI, MVI and lower SLB values, in comparison with the oleogel-EC-based emulsions $(\mathrm{p}<0.05)$. Therefore, sample $\mathrm{K}$ was characterized by the most elastic strength and macroscopic viscosity. The SLB values were below 0.5 , thus the tested emulsions generally presented gel behaviour. There were no statistically significant differences between the computed determinants for the emulsions with EC-oleogels (Figs 5-7), which means that the concentration of ethyl cellulose had no significant impact on the microrheological properties of the $\mathrm{O} / \mathrm{W}$ emulsions.

Liu et al. (2020) indicated that the MS-DWS method allows for an analysis of the microrheological characteristics changes in emulsions in the context of the use of different types and concentrations of the food-grade additives, especially stabilizers. The emulsion with the addition of monogliceryde had the highest elastic strength and the greater macroscopic viscosity. Tisserand et al. (2011) proved that the viscoelastic properties of the emulsion significantly depend on the type and concentration of the added polymers, e.g. alginate, xanthan, carrageenan. If the polymer content increased, both the elasticity and viscosity values became higher. 


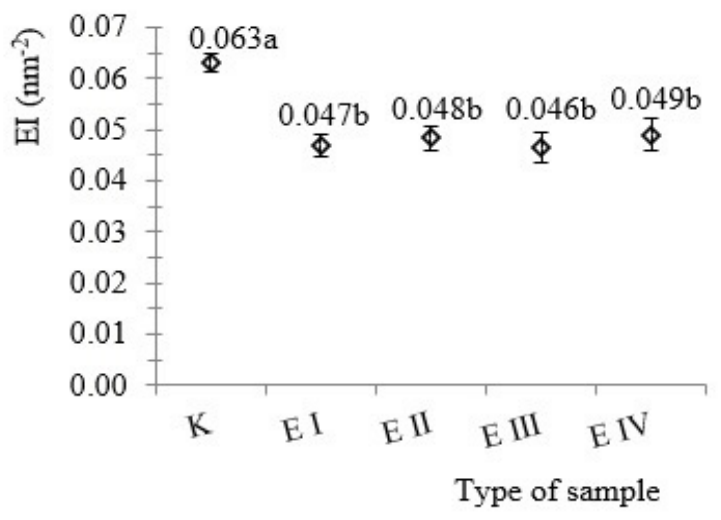

Fig. 5. Elasticity index (EI) of emulsions based on anhydrous milk fat or oleogels with EC content. a, b, c-different letters mean statistically significant differences.

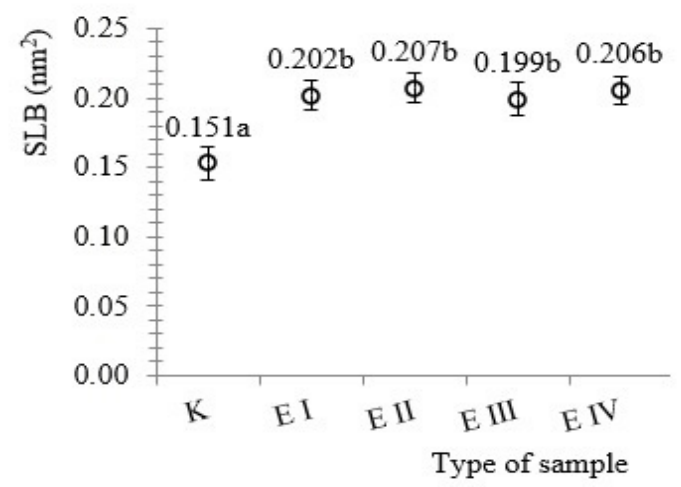

Fig. 6. Solid-liquid balance (SLB) of emulsions based on anhydrous milk fat or oleogels with EC content. a, b, c-different letters mean statistically significant differences.

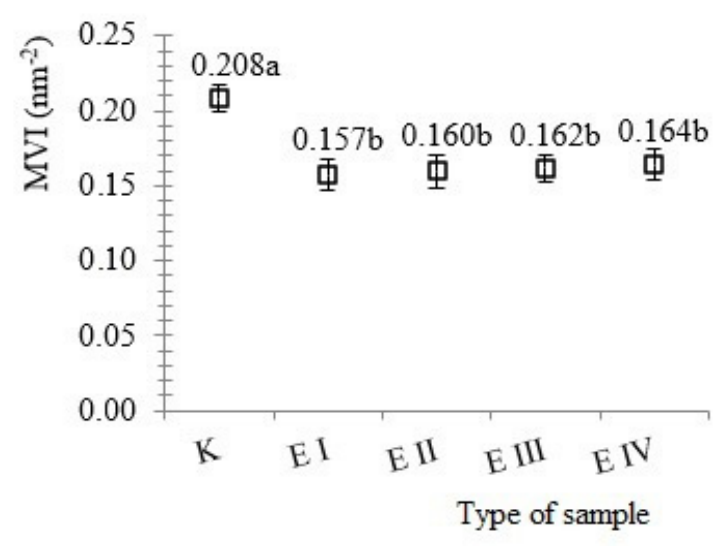

Fig. 7. Macroscopic viscosity index (MVI) of emulsions based on anhydrous milk fat or oleogels with EC content. a, b, c-different letters mean statistically significant differences.
Szymańska et al. (2020) studied O/W emulsions based on rapeseed oil, the thermal stability of which decreased (to $94.4 \%$ ) due to an increased amount of the addition of marine algae protein preparation. Therefore, it was confirmed that the composition of the emulsion has a significant influence on the stability of the structure at high temperatures (aggressive thermal conditions).

The obtained dispersion systems were subjected to a thermal test $\left(100^{\circ} \mathrm{C}, 30 \mathrm{~min}\right)$. Only slight changes in the integrity of all systems were observed. Samples were characterized by the release of a slight amount of liquid lipid fraction at the surface. However, the determined values of thermal stability remained above $99 \%$ for each variant, which proved that the emulsions were equally resistant to the given thermal test conditions. In addition, model emulsions were analysed in a centrifugal test $\left(3000 \mathrm{r} \mathrm{min}^{-1}\right.$, $30 \mathrm{~min}$ ). It was stated that all variants were stable under such an impact of centrifugal force - no changes in the structure of the samples were observed (Table 2). It was demonstrated that novel low-fat $\mathrm{O} / \mathrm{W}$ emulsions based on ethyl cellulose oleogels showed equally high centrifugal or thermal stability compared to the emulsions obtained with an $\mathrm{AMF}$ content.

The emulsions were analysed in a storage test at $4 / 20 / 40^{\circ} \mathrm{C}$ for 28 days, using a LUMiSizer dispersion analyser. The emulsions stored under refrigerated conditions $\left(4^{\circ} \mathrm{C}\right)$ were characterized by a greatest stability 1 day after their production. Emulsion E I had a virtually unchanged physical stability over 14 days of storage. The destabilization of all low-fat emulsions occurred with the most intensity at $40^{\circ} \mathrm{C}$ between 21 and 28 days from their preparation. Emulsions E I and E II were characterized by the lowest values of instability indexes throughout the entire storage period. In addition, it was observed that in the LUMiSizer test, samples K and E IV showed the worst storage stability at $40^{\circ} \mathrm{C}$ (Fig. 8). In Śliwowska's research (2018), the application of a storage temperature equal to 4 or $20^{\circ} \mathrm{C}$ did not result in statistically significant differences between the mean values of the dispersion system stability rates. However, it was indicated that the emulsions stored at $45^{\circ} \mathrm{C}$ for 14 days had been destabilized.

The stability deterioration of the emulsions based on oleogels stored at $20^{\circ} \mathrm{C}$ began 21 days after they were made. Only in the control sample, the instability phenomena already appeared after the 7th day of storage (Fig. 8).

Taking into account the fact that all of the tested emulsions were characterized by high thermal stability at $100^{\circ} \mathrm{C}$ (Table 2), it may be assumed that the centrifugal force $\left(4000 \mathrm{r} \mathrm{min}^{-1}\right)$ had the greatest impact on the destabilization of the sample structure. Stortz and Marangoni (2014), and O'Sullivan et al. (2016) proved that EC-based oleogels are shear sensitive, and that it probably causes a disruption of the polymer network structure. Extending the storage time of the emulsion at an elevated temperature $\left(40^{\circ} \mathrm{C}\right)$, conducive to destabilization could have contributed to a decrease in the emulsion structure resistance to mechanical factors. 
Table 2. Centrifugal stability and thermal stability of model emulsions

\begin{tabular}{lccccc}
\hline & \multicolumn{5}{c}{ Type of sample } \\
\cline { 2 - 6 } & $\mathrm{K}$ & $\mathrm{E} \mathrm{I}$ & E II & E III & E IV \\
\hline $\begin{array}{l}\text { Centrifugal } \\
\text { stability }\end{array}$ & & & Stable & & $99.8 \pm 0.3 \%$ \\
$\begin{array}{l}\text { Thermal } \\
\text { stability }\end{array}$ & $99.4 \pm 0.2 \%$ & $99.3 \pm 0.3 \%$ & $99.4 \pm 0.2 \%$ & $99.8 \pm 0.3 \%$ & 9 \\
\hline
\end{tabular}
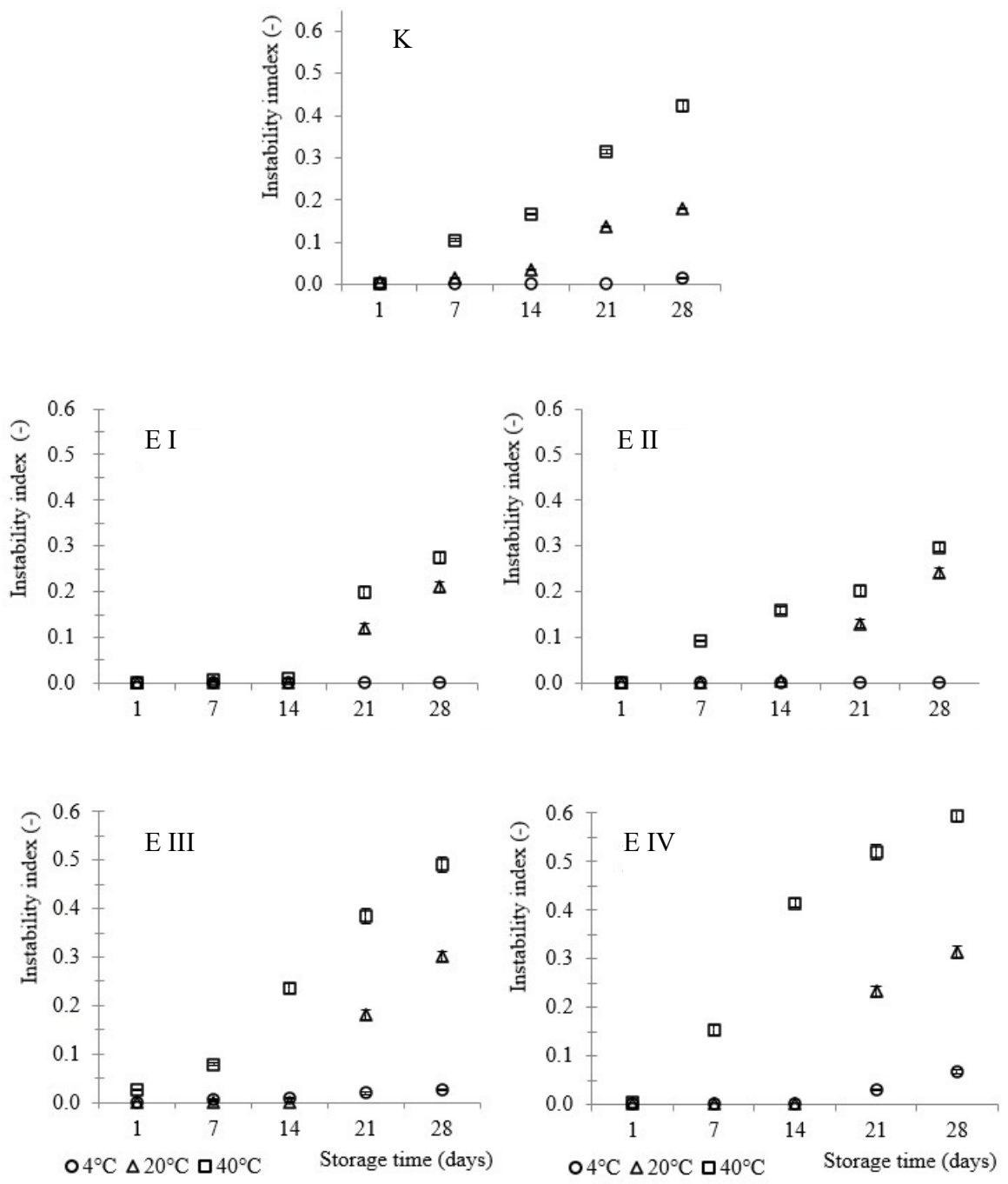

Fig. 8. Instability indexes of emulsions with AMF or EC-oleogels after 1, 7, 14, 21 or 28 days of storage at 4,20 or $40^{\circ} \mathrm{C}$.

Therefore, despite a $6 \%$ concentration of $\mathrm{EC}$, which resulted in the most compact and durable structure, a larger amount of polymer in the oleogel may have been destroyed under the test conditions used.

It was demonstrated that both temperature and storage time had a statistically significant impact on changes in the physical stability of the model emulsions over time. The average values of the instability index increased with decreasing system integrity. The higher temperature and longer storage time had the effect of lowering the emulsion stability (higher instability index). Kowalska et al. (2018) also demonstrated an increase in the coefficient of instability of the $\mathrm{O} / \mathrm{W}$ emulsion with fat esterification as a function of time.

Emulsions based on EC-oleogels, and emulsions with $\mathrm{AMF}$, stored at $4,20,40^{\circ} \mathrm{C}$ for 28 days and analysed using a LUMiSizer test retained their structural integrity. Based on the extinction profiles ("fingerprints") and macroscopic appearance of the samples after the LUMiSizer test, it was found that in the emulsion only reversible separation 

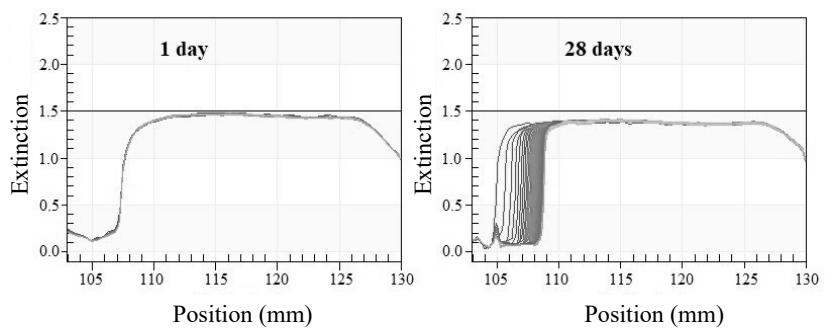

Fig. 9. Extinction profiles of emulsions with AMF after 1 day and 28 days of storage at $20^{\circ} \mathrm{C}$.
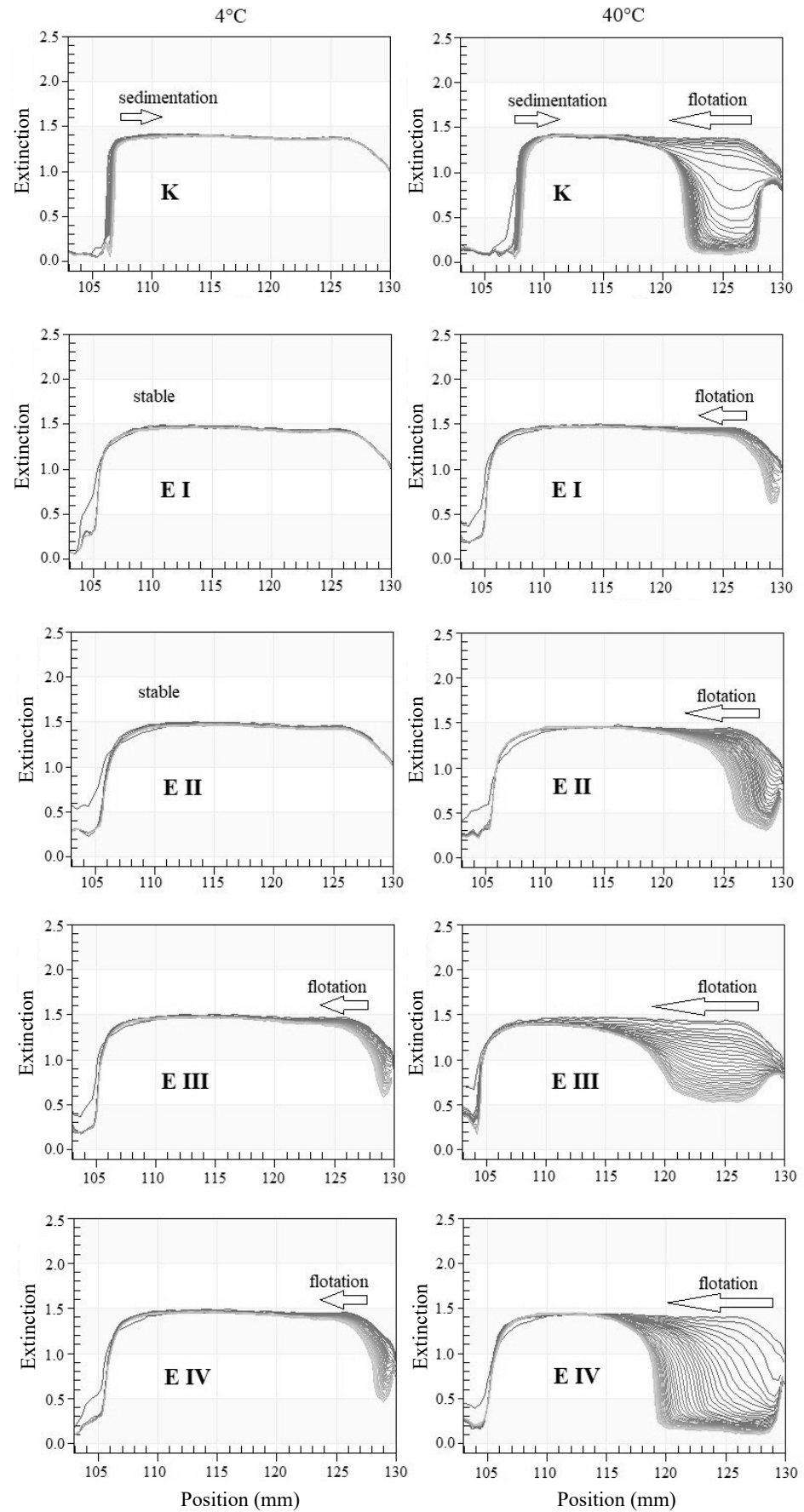

Fig. 10. Extinction profiles of emulsions with AMF or EC-oleogels after 28 days of storage at 4 or $40^{\circ} \mathrm{C}$.
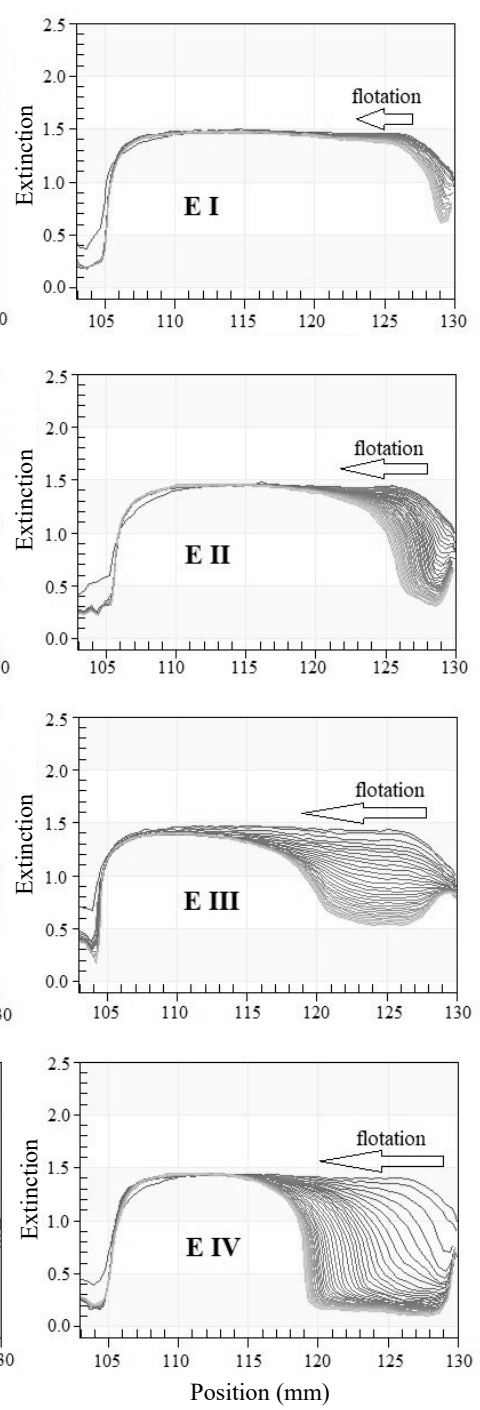

phenomena (flotation/sedimentation) and/or the possible aggregation of particles occurred (Figs 9-11). In turn, Szymańska et al. (2020) indicated that low-fat emulsions, with the addition of algae protein concentrate, stored at $40^{\circ} \mathrm{C}$, were the least stable. The samples were irreversibly destabilized - either the aqueous phase or the oil phase separated from the dispersion system.

Kowalska et al. (2017) and Śliwowska (2018) proved that the backscattered light intensity received (BS) in the height of the whole sample not only indicated a change in the overall stability of the emulsion, but also the mean

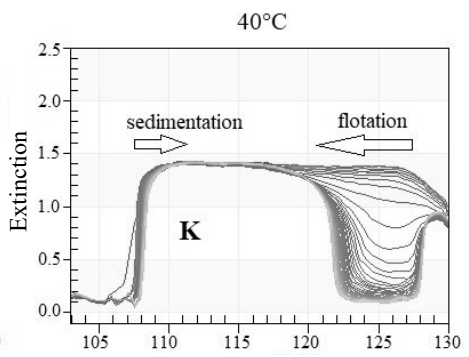



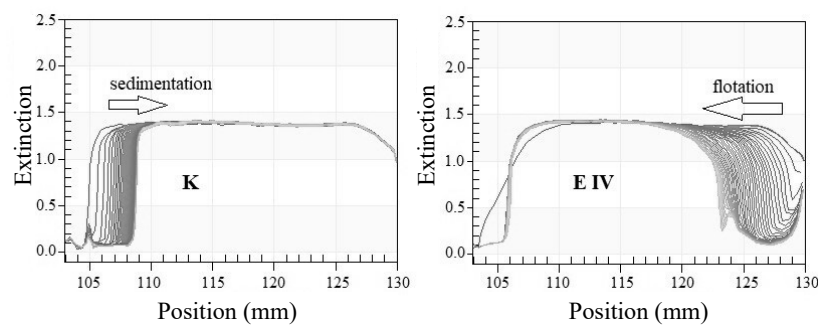

Fig. 11. Extinction profiles of emulsions based on AMF or oleogel with $6 \%$ of EC after 28 days of storage at $20^{\circ} \mathrm{C}$.

average particle size. The extinction of storage time caused the value of the BS parameter to decrease, in comparison with the reference measurement (on day " 0 "). If $\triangle \mathrm{BS}$ was more than $2 \%$, it was considered that the emulsion particle size increased. Therefore, the extinction profiles of the control emulsion after 1-day and 28-days storage were analysed. It was observed that the curves determined after 28 days are located lower on the graph than the curves after 1 day of storage (Fig. 9). In the LUMiSizer test there is no indicator similar to $\triangle \mathrm{BS}$, therefore this difference was not expressed numerically. Accordingly, the possibility of increasing the average model emulsion particle size over time has been found.

Figure 10 presents the extinction profiles of the emulsions stored for 28 days at 4 or $40^{\circ} \mathrm{C}$. Samples EC 4.5 and EC 5.0 were stable throughout the storage period at $4^{\circ} \mathrm{C}$. In turn, in the emulsion with $\mathrm{AMF}$ a sedimentation phenomenon (movement of particles towards the bottom of the tube) was noted, while in EC 5.0 and EC 6.0 - the phenomenon of flotation (movement of particles towards the surface of the sample) occurred. It was found that the velocity of particle separation was more than 3 times higher, compared to the control sample (Table 3). The emulsion with AMF, stored at $20^{\circ} \mathrm{C}$ for 28 days, was subjected to the typical phenomenon of sedimentation (Fig. 11). It was observed that the flotation velocities for EC 5.0, EC 5.5 and EC 6.0 samples after 28 days at $20^{\circ} \mathrm{C}$ were similar (just over $3 \mu \mathrm{m} \mathrm{s}^{-1}$ ) (Table 3 ). The greater the amount of EC in the system, which was stored at $40^{\circ} \mathrm{C}$, the more intense the centrifugal separation and thus the higher the flotation rate of the particles. Of all the tested variants, only the emulsion $\mathrm{K}$ which thermostated at $40^{\circ} \mathrm{C}$ was subject to both flotation and sedimentation (Fig. 10), however, this second phenomenon occurred 10 times slower than the first one (Table 3).

The study, which applied an accelerated analysis of emulsion stability using a LUMiSizer analyser, indicated that changes in particle migration velocity in the centrifugation field were correlated with the values of dispersion instability indexes. Longer storage periods and higher storage temperatures contributed to a lower sample durability and an increase in the velocity of particle sedimentation/ flotation. Depending on the scale of the detected stability
Table 3. Separation velocity $\left(\mu \mathrm{m} \mathrm{s}^{-1}\right)$ of emulsions after 28 days of storage (position in range of $125-130 \mathrm{~mm}$ for flotation and 106$110 \mathrm{~mm}$ for sedimentation)

\begin{tabular}{clllll}
\hline $\begin{array}{c}\text { Tempera- } \\
\text { ture of } \\
\text { storage } \\
\left({ }^{\circ} \mathrm{C}\right)\end{array}$ & K & E I & E II & E III & E IV \\
\cline { 2 - 6 } 4 & S 0.19 & - & - & F 0.64 & F 0.87 \\
20 & S 1.15 & F 2.11 & F 3.05 & F 3.13 & F 3.08 \\
40 & S 0.42 & F 2.91 & F 3.75 & F 5.44 & F 7.36 \\
\hline
\end{tabular}

$\mathrm{S}$ - sedimentation, $\mathrm{F}$ - flotation.

variations, this may indicate the initiation/progress of dispersed phase particle aggregation. Ullmann et al. (2017) and Zielińska (2018) presented similar statements.

The results of this study prove that modern methods, i.e. the MS-DWS method and/or CSA method, are useful for assessing the variations in the physical stability of oil-in-water emulsions obtained using unconventional components.

Multidimensional statistical analysis (cluster analysis) was performed to isolate groups of model emulsions which were similar in terms of selected physical parameters: centrifugal stability, thermal stability, EI, SLB, MVI, instability indexes at 4,20 or $40^{\circ} \mathrm{C}$, separation velocity at 4 or $40^{\circ} \mathrm{C}$. There were 2 groups (clusters) of emulsion, the binding distance was: $\mathrm{y}=0.3517$ (Fig. 12). Cluster 1 includes emulsions K, E I and E II, while cluster 2 includes samples E III and E IV. Both emulsion groups presented comparable mean centrifugal or thermal stability. Cluster 1 was characterized by higher average values of EI and MVI, however, the SLB, instability indexes and separation velocities were lower, compared to cluster 2. Such indicator values may result in the high stability of dispersions. On the basis of the coefficients of variation determined for each cluster, it was found that the instability indexes of the emulsions stored at $4{ }^{\circ} \mathrm{C}$ were the most varied (approximately 43 and $65 \%$

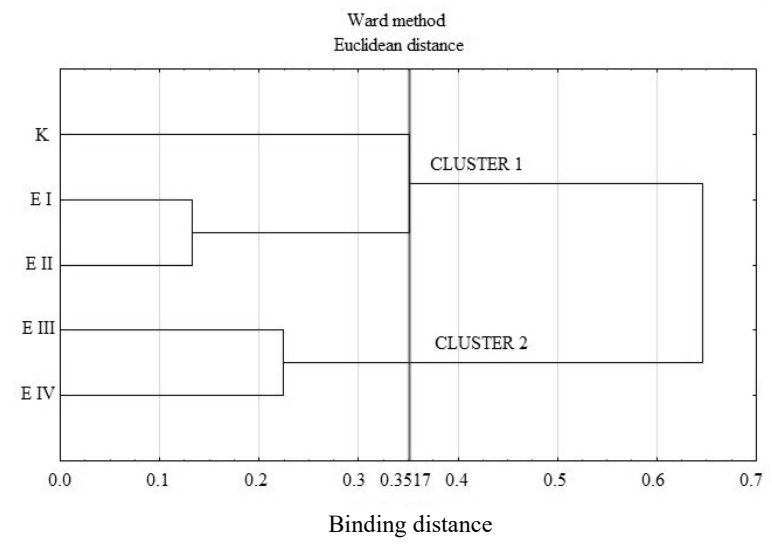

Fig. 12. Tree diagram as a result of cluster analysis using the Ward method. 
respectively). In addition, the coefficient of variation for cluster 1 was found to be approximately $45 \%$ in terms of the separation velocity of emulsions stored at $20^{\circ} \mathrm{C}$.

\section{CONCLUSIONS}

1. Oleogels with ethyl cellulose, except for ethyl cellulose, exhibited a higher centrifugal stability, compared to anhydrous milk fat. However, the anhydrous milk fat was characterized by greater values of textural parameters.

2. Oleogels based on rapeseed oil with ethyl cellulose may be used as the fat phase of novel low-fat oil-in-water emulsions. The emulsions with ethyl cellulose-oleogels did not differ from each other in their microrheological determinant values.

3. All of the model emulsions demonstrated a high stability in centrifugal/thermal tests. Both temperature and time of storage had a significant influence on the deterioration of the physical stability of the emulsions with anhydrous milk fat or ethyl cellulose-oleogels.

4. The emulsions with ethyl cellulose (EC 4.5 and 5.0) presented the highest degree of physical durability at various storage temperatures as a function of time. Instability phenomena, which occurred in all emulsions, were reversible (sedimentation and/or flotation). The emulsions thermostated at $40^{\circ} \mathrm{C}$ caused an acceleration in the occurrence of destabilization, compared to storage at 4 or $20^{\circ} \mathrm{C}$.

5. Ethyl cellulose is an effective edible oil structuring agent, however, it is difficult to use due to the high temperature of the oleogelation process.

6. The possibility of using the Centrifugal Stability Analysis (CSA) method and Multi Speckle Diffusing Wave Spectroscopy (MS-DWS) method in determining the physical stability of innovative oleogel-in-water emulsions has been demonstrated.

Conflict of interest: The Authors do not declare conflict of interest.

\section{REFERENCES}

Co E.D. and Marangoni A.G., 2012. Oleogels: an alternative edible oil-structuring method. J. Am. Oil Chemists' Society, 89, 749-780. https://doi.org/10.1007/s11746-012-2049-3

Dybkowska E., 2015. The role of fatty acids in nutrition and human health (in Polish). In: The importance of rational nutrition in health education (Ed A. Wolska-Adamczyk). WSiZ Publishing House, Warsaw, Poland.

EFSA (European Food Safety Authority), 2017. Dietary reference values for nutrients: Summary report. EFSA supporting publication. https://doi.org/10.2903/sp.efsa.2017.e15121

Ferreira-Dias S., Osório N.M., Rodrigues J., and Tecelão C., 2019. Structured lipids for foods. In: Encyclopedia of Food Chemistry (Eds L. Melton, F. Shahidi, P. Varelis). Elsevier, 3, 357-369.

https://doi.org/10.1016/b978-0-08-100596-5.21766-6
Gravelle A., Barbut S., and Marangoni A.G., 2013. Fractionation of ethylcellulose oleogels during setting. Food Function 4, 153-161. https://doi.org/10.1039/c2fo30227f

Hughes N.E., Marangoni A.G., Wright A.J., Rogers M.A., and Rush J.W., 2009. Potential food applications of edible oil organogels. Trends Food Sci. Technol., 20, 470-480. https:// doi.org/10.1016/j.tifs.2009.06.002

Kowalska M., Krzton-Maziopa A., Żbikowska A., and Tarnowska K., 2017. Rheological properties and physical stability of $\mathrm{O} / \mathrm{W}$ emulsions stabilized by diacylglycerols formed during enzymatic interesterification. Applied Rheology, 27, 1-9.

Kowalska M., Woźniak M., Krzton-Maziopa A., Tavernier S., Pazdur L., and Żbikowska A., 2018. Development of the emulsions containing modified fats formed via enzymatic interesterification catalyzed by specific lipase with various amount of water. J. Dispersion Sci. Technol., https://doi.org /10.1080/01932691.2018.1467328

Kowalska M. and Żbikowska A., 2016. Study of stability of sesame oil-in-water emulsions determined using an optical analyzer and measurement of particle size and distribution. J. Dispersion Sci. Technol., 37, 10, 1408-1414. https://doi. org/10.1080/01932691.2015.1111143

Kowalska M., Żbikowska A., Śmiechowski K., and MarciniakLukasiak K., 2014. Effect of sunflower lecithin quantity and homogenization time on stability of food emulsion containing walnut Oil. Food. Sci. Technol. Qual., 92, 78-91. https://doi.org/10.15193/zntj/2014/92/078-091

Laredo T., Barbut S., and Marangoni A.G., 2011. Molecular interactions of polymer oleogelation. Soft Matter 7, 27342743. https://doi.org/10.1039/c0sm00885k

Lindman S., 2014. The effect of rapeseed oil and palm oil supplement and milking frequency on milk yield and milk fat quality. Swedish University of Agricultural Sciences, Uppsala, Sweden.

Liu Y., Wei Z.C., Deng Y.Y., Dong H., Zhang Y., Tang X.J., Li P., Liu G., and Zhang M.W., 2020. Comparison of the effects of different food-grade emulsifiers on the properties and stability of a casein-maltodextrin-soybean oil compound emulsion. Molecules, 25(3), 458. https://doi.org/ 10.3390/molecules 25030458

Lupi F.R, Gabriele D., de Cindio B., Sanchez M.C., and Gallegos C., 2011. A rheological analysis of structured water-in-olive oil emulsions. J. Food Eng., 107, 296-303. https://doi.org/10.1016/j.jfoodeng.2011.07.013

Öğütcü M. and Yılmaz E., 2014. Oleogels of virgin olive oil with carnauba wax and monoglyceride as spreadable products. Int. J. Fats Oils, 65, 3, 1-11. https://doi.org/10.3989/ gya.0349141

Öğütcü M., Arifoğlu N., and Yılmaz E., 2015. Preparation and characterization of virgin olive oil-beeswax oleogel emulsion products. J. Am. Oil Chemists' Society, 92, 459-471. https://doi.org/10.1007/s11746-015-2615-6

Onacik-Gür S., and Żbikowska A., 2019. Effect of high-oleic rapeseed oil oleogels on the quality of short-dough biscuits and fat migration. J. Food Sci. Technol., 1-10. https://doi. org/10.1007/s13197-019-04193-8

Onacik-Gür S., Żbikowska A., Przybysz M., and Kowalska M., 2017. Assessment of physical properties of structured oils and palm fat. Materiale Plastice, 54, 4, 800-805. https:// doi.org/10.37358/mp.17.4.4949 
Orsavova J., Misurcova L., Ambrozova J.V., Vicha R., and Mlcek J., 2015. Fatty acids composition of vegetable oils and its contribution to dietary energy intake and dependence of cardiovascular mortality on dietary intake of fatty acids. Int. J. Molecular Sci., 16, 12871-12890. https://doi. org/10.3390/ijms160612871

O'Sullivan C.M., Barbut S., and Marangoni A.G., 2016. Edible oleogels for the oral delivery of lipid soluble molecules: Composition and structural design considerations. Trends in Food Sci. Technol., 57, 59-73. https://doi.org/10.1016/j. tifs.2016.08.018

Pasqua A., Fleury M., Brun A., Cristiano M.C., and Cosco D., 2014. Potential application of Micro-Rheology-Rheolaser Lab® in food sciences. In: Advanced Technologies in Food Science I - Innovative techniques for food analysis, characterization and quality control (Ed. Andrew J.S. Coats). Biomed Research International, 6, 1, 60-69.

Piotrowska A. and Waszkiewicz-Robak B., 2007. Measurement of light backscattering intensity with a new method of liquid dispersion stability evaluation in food. Technol. Progr. Food Process, 2, 26-28.

Stortz T.A., and Marangoni A.G., 2011. Heat resistant chocolate. Trends in Food Sci. Technol., 22(5): 201-214. https:// doi.org/10.1016/j.tifs.2011.02.001

Stortz T.A., Zetzl A.K., Barbut S., Cattaruzza A., and Marangoni A.G., 2012. Edible oleogels in food products to help maximize health benefits and improve nutritional profiles. Lipid Technol., 24(7): 151-154. https://doi. org/10.1002/lite.201200205

Szymańska I., Żbikowska A., and Marciniak-Lukasiak K., 2020. Effect of addition of a marine algae (Chlorella protothecoides) protein preparation on stability of model emulsion systems. J. Dispersion Sci. Technol., 41(5): 699-707. https://doi.org/10.1080/01932691.2019.1611438
Śliwowska A., 2018. Evaluation of physicochemical and application properties of cosmetic formulations containing jasmonates together with assessment of kinetics of their diffusion through skin imitating barriers. Ph.D. Thesis, Adam Mickiewicz University in Poznań, Poland.

Tisserand C., Kotzev A., Fleury M., Brunel L., Bru P., and Meunier G., 2011. Non-contact measurement of viscoelastic properties of biopolymers. NSTI-Nanotech, 1, 36-40

Ullmann C., Babick F., Koeber R., and Stintz M., 2017. Performance of analytical centrifugation for the particle size analysis of real-world materials. Powder Technol., 319, 261-270. https://doi.org/10.1016/j.powtec.2017.06.057

Xu D., Qi Y., Wang X., Li X., Wang S., Cao Y., Wang C., Sun B., Decker E., and Panya A., 2017. The influence of flaxseed gum on the microrheological properties and physicochemical stability of whey protein stabilized $\beta$-carotene emulsions. Food Funct., 8, 415-423. https://doi.org/10.1039/ c6fo0 $01357 \mathrm{k}$

Zetzl A.K., Marangoni A.G., and Barbut S., 2012. Mechanical properties of ethylcellulose oleogels and their potential for saturated fat reduction in frankfurters. Food Function, 3, 327-337. https://doi.org/10.1039/c2fo10202a

Zielińska A., 2018. Synthesis and characterization of solid lipid nanoparticles and nanostructured lipid carriers dedicated to cosmetic and pharmaceutical applications. Ph.D. Thesis, Adam Mickiewicz University in Poznań, Poland.

Żbikowska A., Kupiec M., Marciniak-Lukasiak K., and Kowalska M., 2017. Oleogels - perspectives on applying them to food. Żywność, Nauka, Technologia, Jakość, 24, 3, 112, 5-13.

Żbikowska A., Kupiec M., and Kowalska M., 2019. Comparison of oleogels properties obtained with different structureforming substances. Polish J. Natural Sci., 34, 2, 273-284. 